\title{
The idea of mismatch in evolutionary medicine
}

\begin{abstract}
Mismatch is a prominent concept in evolutionary medicine and a number of philosophers have published analyses of this concept. The word 'mismatch' has been used in a diversity of ways across a range of sciences, leading these authors to regard it as a vague concept in need of philosophical clarification. Here, in contrast, we concentrate on the use of mismatch in modelling and experimentation in evolutionary medicine. This reveals a rigorous theory of mismatch within which the term 'mismatch' is indeed used in several ways, not because it is ill-defined but because different forms of mismatch are distinguished within the theory. Contemporary evolutionary medicine has unified the idea of 'evolutionary mismatch', derived from the older idea of 'adaptive lag' in evolution, with ideas about mismatch in development and physiology derived from the Developmental Origins of Health and Disease (DOHaD) paradigm. A number of publications in evolutionary medicine have tried to make this theoretical framework explicit. We build on these to present the theory in as simple and general a form as possible. We introduce terminology, largely drawn from the existing literature, to distinguish the different forms of mismatch. This integrative theory of mismatch captures how organisms track environments across space and time on multiple scales in order to maintain an adaptive match to the environment, and how failures of adaptive tracking lead to disease. Mismatch is a productive organising concept within this theory which helps researchers articulate how physiology, development and evolution interact with one another and with environmental change to explain health outcomes.
\end{abstract}

\section{The dual origins of mismatch ${ }^{1}$}

The capacity of organisms to adapt to their environments is sometimes outpaced by the speed and scale of environmental change. The likelihood of 'adaptive lag', in which evolution by natural selection fails to keep pace with a changing selective environment, was widely accepted by the founders of the modern synthesis (Dobzhansky [1963]). It offered a plausible explanation of many cases of observed maladaptation. Mayr referred to extreme cases, in which organisms are unable to adapt to changed conditions, as "evolutionary traps" (Mayr [1942], pp. 224-5).

This phenomenon was understood to result from both spatial and temporal changes in environment. An increased incidence of disorders resulting from lactose intolerance in a population with Han Chinese ancestry could result either from increased consumption of milk products over time or from migration to an environment in which milk products are an important source of nutrition. Throughout this paper 'environmental change' should be read as including both temporal and spatial change.

The idea of adaptive lag was prominent in thinking about human health well before the emergence of evolutionary medicine as it is now understood (Williams and Nesse [1991]; Nesse and Williams [1994]). As early as 1922 one author claimed to explain poor mental health with a 'Theory of the Cave Man in the Modern City' (Ogburn [1922], p. 284ff). In the 1950s human geneticist Neel initiated the study of ' $[t]$ he genetic significance of changing dietary patterns'. Neel's primary motivation was the opportunity to study natural selection in the human species. ${ }^{2}$ But he was aware of the medical implications when he argued that high levels of dietary fat and salt in modern environments impose novel selection pressures on human metabolism, leading to reduced reproductive fitness. He suggested that European populations may have partially adapted to these novel diets, and that the rapid modernisation in other parts of the world might have more severe health consequences (Neel [1958]).

\footnotetext{
${ }^{1}$ Supplementary materials containing the data on which this historical introduction is based - keyword literature search results and a summary diagram showing cross-citation between key authors can be found at:

${ }^{2}$ Neel begins his paper on the genetic significance of changing dietary patterns: "although in recent years the mathematical papers of Fisher, Wright, Haldane and others have greatly advanced our understanding of the manner in which the changes in gene frequency which constitute biological evolution come about, our knowledge of the actual workings of natural selection in human populations is almost nil..." ((Neel [1958], p. 43) See also his late-life reflections on this work, (Neel [1989], p. 811)).
} 
In the late 1960s the influential psychologist Bowlby introduced the term "environment of evolutionary adaptedness" and argued that, "[w]e can ... be fairly sure that none of the environments in which civilised, or even half-civilised, man lives today conforms to the environment in which man's environmentally stable behavioural systems were evolved and to which they are intrinsically adapted." (Bowlby [1969], p. 59).

The term 'mismatch' was first used to label the phenomenon of adaptive lag in 1988 (Eaton et al. [1988], p. 739). It was used in the same sense in Williams and Nesse's influential 1991 paper 'The Dawn of Darwinian Medicine' ([1991], p. 9) and their book Why We Get Sick (Nesse and Williams [1994]). In a recent evolutionary medicine textbook, we learn that "[m]ismatch occurs when organisms that were well adapted to one set of environmental circumstances cannot evolve rapidly enough to adapt to a new set of circumstances." (Stearns and Medzhitov [2015], p. 13) The lecture series which accompanies this textbook links 'mismatch' to the older name of adaptive lag: 'Adaptation is rarely precise and often lags' (Stearns [2015], p. 1.42). Behavioral ecologists Laland and Brown also use 'mismatch' and 'adaptive lag' as synonyms: "it is a truism that all organisms must experience some adaptive lag, here meaning a mismatch between current selection pressures and behavior" ([2006], p. 98). Following Riggs ([1993], p. 1289) we refer to this mismatch between genes and environment due to adaptive lag as evolutionary mismatch.

A decade after the term 'mismatch' was adopted in evolutionary medicine a distinction began to be drawn between this evolutionary mismatch between genes and environment and the "mismatch between body and environment" (Bateson [2001], p. 932) which results from failures of phenotypic plasticity. ${ }^{3}$ This idea emerged from work on the 'Developmental Origins of Health and Disease' (DOHaD), an area of biomedical research which studies how events early in the lifecourse influence later health outcomes (Peter D. Gluckman et al. [2016]). Following Kuzawa ([2008]) we refer to this as 'developmental mismatch' (see also Peter D. Gluckman et al. [2009], p. 204).

Type II diabetes was a prominent example in these early discussions of mismatch phenomena. The 'thrifty genotype' hypothesis (Neel [1962]) attributed rises in the incidence of Type 2 diabetes to a mismatch between the modern nutritional environment and genes adapted to an ancestral nutritional environment. This was an evolutionary mismatch hypothesis, although the term 'mismatch' was not used to describe it for another twenty-four years. The later 'thrifty phenotype' hypothesis (Hales and Barker [1992]) attributed spikes in the incidence of Type 2 diabetes to the existence of birth cohorts with ill-nourished mothers but lives of nutritional abundance. Barker and collaborators hypothesised that the developing fetus responds to maternal cues of a poor nutritional environment by developing a physiology that is well adapted to survival in those conditions. If, however, the fetus grows up to experience nutritional abundance these physiological setting increase the risk of Type II diabetes, obesity, and other aspects of 'metabolic syndrome'. This hypothesis became paradigmatic of the $\mathrm{DOHaD}$ approach to disease and it has been extensively studied, although many empirical issues remain unresolved. ${ }^{4}$

The first author to describe the thrifty phenotype hypothesis as 'mismatch' between the physiological settings adopted by the fetus and the nutritional environment of the adult was Bateson, a distinguished ethologist with a strong interest in behavioural development (Bateson and Martin [1999], p. 114; Bateson [2001], p. 932). Bateson was interested in applying his ideas about the interaction of development and evolution to the new field of Evolutionary Medicine (Bateson [1997], pp. 8-11). The term 'mismatch' was applied again to DOHaD phenomena in a 2004 manifesto published in Nature (Bateson et al. [2004]). This resulted from a workshop organised by Bateson with behavioural

\footnotetext{
3 “[A] single genotype being able to produce different phenotypes in different environments (phenotypic plasticity)" (Peter D. Gluckman et al. [2005], p. 527)

${ }^{4}$ For an introduction to this research see Gluckman and Hanson ([2004]). For a historical treatment, and the relationship of this work to earlier ideas about fetal health, see Richardson ([2021]). As pointed out by an anonymous referee, our approach being descriptive, it does not consider the social and ethical implications of these DOHaD mismatch hypotheses - see Sharp, Schellhas, Richardson and Lawlor ([2019]).
} 
ecologists, evolutionary biologists (particularly experts on the evolution of phenotypic plasticity) and leading $\mathrm{DOHaD}$ researchers, including Barker, originator of the thrifty phenotype hypothesis, and Gluckman, one of its most influential advocates. After this, the term spread rapidly via high-profile publications in the DOHaD literature (Peter D Gluckman and Hanson [2004]; Peter D. Gluckman and Hanson [2004a]; Peter D. Gluckman et al. [2005]; Peter D Gluckman et al. [2005]) and books explaining the DOHaD paradigm to a wider audience (Peter D. Gluckman and Hanson [2004b]; Gluckman and Hanson [2006]).

Historian of science Buklijas, writing with leading DOHaD researchers Gluckman and Hanson, describe this key phase as follows, "In the early 2000s, the Cambridge ethologist Patrick Bateson, first alone [65] and then together with the authors and others [66], proposed a comprehensive hypothesis that placed the $\mathrm{DOHaD}$ phenomenon firmly within the evolutionary framework of developmental plasticity" (Peter D. Gluckman et al. [2016], p. 6 Ref. 65 is Bateson ([2001]); Ref. 66 is Bateson et al ([2004])).

The existence of two different 'mismatch' hypotheses to explain Type II diabetes, necessitated terminology to mark the difference between them. Bateson contrasted mismatch between gene and environment (our 'evolutionary mismatch') with "mismatch between body and environment" (Bateson [2001], p. 932). But Kuzawa ([2008]) called this second kind of mismatch 'developmental mismatch', as did Gluckman et al in Principles of Evolutionary Medicine (Peter D. Gluckman et al. [2009]). We follow this usage in the remainder of the paper.

It soon became clear to these researchers that there are several different ways in which an organism can fail to update its phenotype to match a changing environment. Kuzawa pictures this as a continuum of timescales (Figure 1) and a series of mechanisms that produce, or fail to produce, an adaptive match between phenotype and environment on different timescales. Adaptive evolution by natural selection is the slowest of these processes and homeostatic adaptation is the fastest. The failure of any of these mechanisms can produce a mismatch between the organism and its environment.

Cycle duration Adaptation

\begin{tabular}{|c|c|c|c|}
\hline Years & & Mode & Process \\
\hline 0.00000001 & Seconds & \multirow{4}{*}{ Physiological } & \\
\hline 0.0001 & Hours & & Homeostasis \\
\hline 0.001 & Days & & \\
\hline 0.1 & Months & & Allostasis \\
\hline 1 & Years & \multirow{3}{*}{$\begin{array}{l}\text { Developmental } \\
\text { Intergenerational }\end{array}$} & Plasticity \\
\hline 10 & Decades & & Inertia \\
\hline 100 & Centuries & & \\
\hline 1000 & Millennia & \multirow[t]{2}{*}{ Genetic } & \multirow[t]{2}{*}{ Natural Selection } \\
\hline 1000000 & Millions & & \\
\hline
\end{tabular}

Figure 1. Kuzawa's representation of adaptation on multiple timescales. The right side shows timescales on which organisms experience "ecological change." The left side shows corresponding "mode of adaptation" by which organisms can track change on these timescales. "Inertia" is Kuzawa's term for epigenetic inheritance across two or more generations (Reproduced from Kuzawa [2008], p. 342).

Other theorists reached the same conclusion. Following the success of the 2004 Nature paper, Gluckman convened a larger and overlapping gathering of "clinicians and public-health specialists from high-income and low-income countries, developmental and evolutionary biologists, geneticists, 
anthropologists, and economists" (P. D. Gluckman et al. [2009], p. 1654). The resultant twentyauthored manifesto 'Towards a new developmental synthesis: adaptive developmental plasticity and human disease' was published in Lancet. It endorsed the idea that organisms use multiple 'modes of adaptation' ${ }^{5}$ to track environments that change simultaneously on multiple timescales (Figure 2).

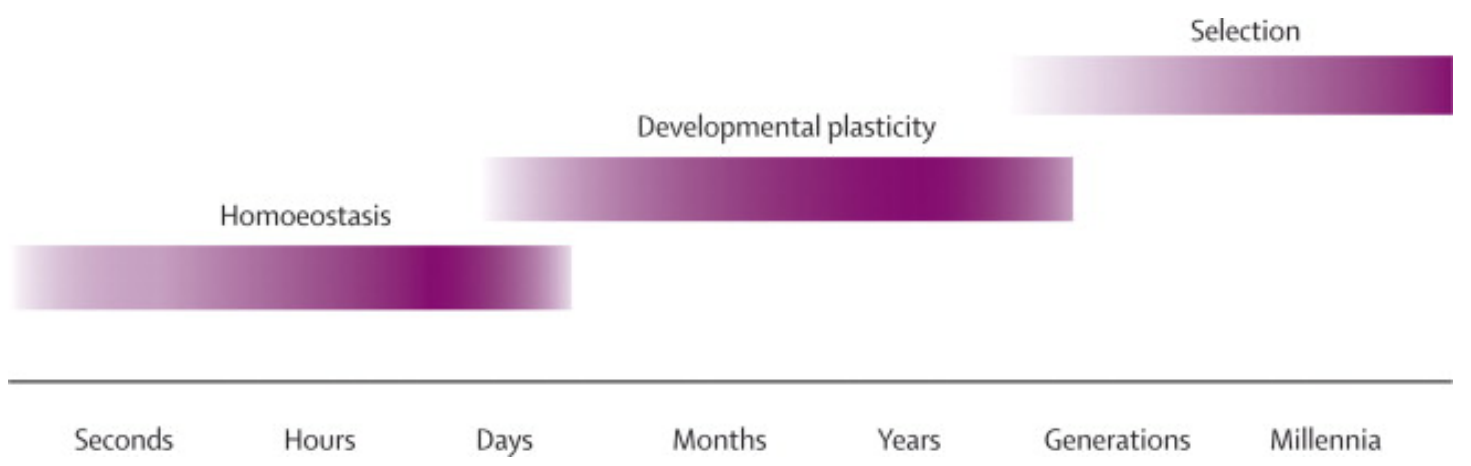

Figure 2. “Modes of Adaptation” (Reproduced from P. D. Gluckman et al. [2009], p. 1655).

The key development in this work was the realisation that organisms can adapt to an environmental challenge either by evolution or by physiological or developmental adaptation, and that these modes of adaptation interact. ${ }^{6}$ This idea is now commonplace in evolutionary medicine. Research on nutrition notes that, "[m]ismatch occurs when the timescale and/or magnitude of environmental change exceeds the combined capacity of adaptation owing to homeostatic mechanisms, phenotypic plasticity and transgenerational adaptation." (Raubenheimer et al. [2012], p. 1641). Research on populations at high altitude notes that, "Knowing how long the population has resided at altitude is important for considering the potential for modes of adaptation that occur on time scales ranging from short (reversible acclimatization), to intermediate (developmental) and long (genetic)." (Beall [2014], p. 253). While introductory presentations of Evolutionary Medicine still emphasise the evolutionary timescale, they acknowledge that mismatch can also be identified on developmental timescales: "evolutionary mismatch, [is] defined here as the phenomenon by which previously adaptive alleles are no longer favoured in a new environment... other uses of mismatch are applied over the life course" (Manus [2018], p. 190).

In contemporary evolutionary medicine we can see this sophisticated theory of mismatch on multiple timescales at work. Just as Bateson hoped, it allows insights to flow in both directions between evolutionary theory and the study of development and physiology. Two examples of this mutual illumination are, first, that the goals ${ }^{7}$ built into mechanisms of homeostasis and allostasis are trade-offs between multiple life-history goals and, second, that mechanisms of physiological and developmental adaptability shape the selection pressures acting on genotypes. These insights are explained more fully in Section 3, using examples from nutritional ecology.

The theory of mismatch contributes to the defining project of evolutionary medicine, which is to analyze and explain susceptibility to disease (Nesse and Williams [1994]). One obvious cause of susceptibility to disease is maladaptation to the environment - an organism that loses an arms race with its parasites,

\footnotetext{
${ }^{6}$ Evolutionary medicine inherits the phrase 'physiological adaptation' from medicine, where it is standard. An anonymous referee has pointed out that this may be confusing to readers of this journal. Introductory material for medical students sometimes points out that physiological adaptation should not be confused with Darwinian adaptation by natural selection ('Physiological adaptation - Definition and Examples - Biology Online Dictionary' [2019]) Here we repeat that warning.

${ }^{7}$ An anonymous referee has asked us to make clear that these are goal-directed processes strictly in the cybernetic sense and do not have intentionality.
} 
or which is unable to obtain the foodstuffs which its digestive system evolved to process, is likely to suffer from pathology. It is for this reason that 'Mismatch' occurs in the lists of 'pathways to disease' that structure evolutionary medicine textbooks (e.g. Peter D. Gluckman et al. [2009] (2nd Ed. 2016); Stearns and Medzhitov [2015]). Mismatch is a way to explain the existence of maladaptive phenotypes within a broadly adaptationist (Godfrey-Smith [2001]) framework which expects that as a result of natural selection both constitutive phenotypes and mechanisms of plasticity will be well-adapted to their environments and that the operation of those mechanisms of plasticity will produce adaptive phenotypes. So mismatch is an important way to explain susceptibility to disease in evolutionary medicine.

\section{How 'mismatch' is used in evolutionary medicine}

It has been argued that, "scientists have generally been working without a clear definition of mismatch." (e.g. Cofnas [2016], pp. 507-8). The impression that mismatch is a vague concept in urgent need of clarification may arise because the word occurs in both scientific and popular works across a wide range of disciplines. But many terms that are used in precise and consistent ways in one scientific discipline are used in other ways in other disciplines and outside science. 'Mismatch' is one of these. The fact that, for example, mismatch theories of emotion regulation (Mandler [1984]) adopt the word 'mismatch' for their own purposes does not show that mismatch is badly defined either in those theories or in evolutionary medicine.

In this section we examine how the vernacular meaning of the word 'mismatch' relates to its use in evolutionary medicine and then discuss that use in more detail. Section 3 is a case-study of this use in one well-developed area of evolutionary medicine, nutritional ecology. In this field mismatch is a clearly defined and productive concept whose role in the research corresponds to the explicit definitions discussed in Section 1. In Section 5 we criticise some previous proposals by philosophers to 'clarify' the concept of mismatch in evolutionary medicine. These do not reflect how mismatch is understood in contemporary evolutionary medicine and as theoretical constructs they are less useful than the understanding of mismatch we have identified there.

\subsection{Vernacular meaning}

In everyday English, a square peg is mismatched to a round hole if our standard of success for pegs is fitting through holes. There is a mismatch between supply and demand if there is too much or too little supply to meet demand, the standard of success in this case being profits. In this commonsense way, we can speak of a 'mismatch' in biology whenever we have a biological unit, an actual environment, a reference environment, and some measure of utility according to which the unit would have higher utility in the reference environment than in its actual environment (Figure 3). The biological unit is commonly a gene, a genotype or an organism, but many other units could potentially be described as 'mismatched'. The reference environment is usually the historical environment to which the unit was adapted. But other choices are possible - Morris ([2018]) suggests assessing mismatch with reference to an optimal environment (see Section 5). The utility function is usually biological fitness or some fitness surrogate (such as energetic efficiency). But other utility functions could be used - Lloyd, Wilson and Sober point to subjective well-being as one alternative (Lloyd et al. [2011], p. 5). Evolutionary medicine researchers writing for a popular audience may well talk of 'mismatch' in cases where environmental change reduces subjective well-being. But that does not mean that they use a vague concept of mismatch in their scientific practice. As we saw in Section 1, explicit definitions of mismatch in evolutionary medicine consistently use biological fitness, and the models, operationalisations and other elements of scientific practice in evolutionary medicine research comply with that definition, as we show in more detail in Section 3. 


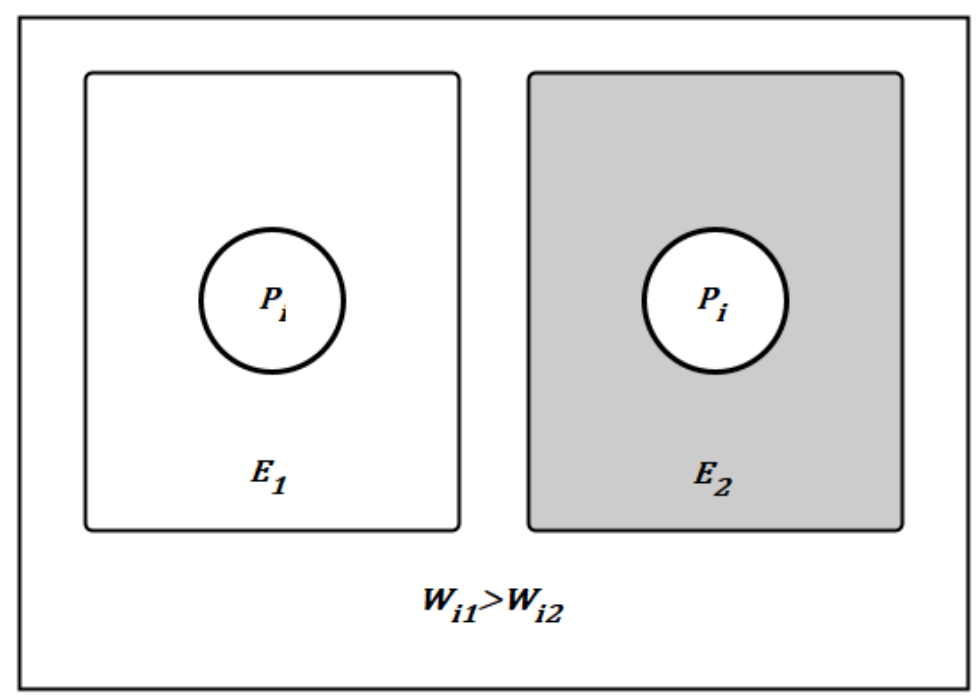

Figure 3. Biological applications of the term 'mismatch' conform to a general schema: A biological unit $i$ with phenotype $P$ is in a state of mismatch with respect to its actual environment $E$ for a given utility function $f$, if considering a set of possible states of the environment $\left[E_{1}, E_{2}, E_{N}\right]$, i performs lower with respect to $f$ in its actual environment, than it would in another state of the possible environment (the reference environment). For instance, in Figure 3, $i$ is in a state of mismatch with respect to $P$ and $E_{2}$ when compared to $E_{1}$ for the fitness function $W\left(W_{i_{1}}>W_{i_{2}}\right)$.

When evolutionary medicine is studying change on evolutionary timescales, the reference environment for mismatch is the historical environment to which the organism is adapted, often referred to as the 'environment of evolutionary adaptedness' or EEA. The EEA concept has been criticised because a species can occupy many different environments in the period over which a trait evolves and also because of niche construction (Buller [2005], pp. 59-60; Laland and Brown [2002], [2006]). The primary target of these criticisms is the use of the EEA concept in evolutionary psychology. According to Buller evolutionary psychologists regard the EEA as a specific set of environmental factors, and this way of thinking can be seen in the first paper to use the term 'mismatch' in evolutionary medicine (Eaton et al. [1988]). However, as can be seen in the models presented in Section 3, the EEA is not normally conceived as a specific set of environmental factors, let alone a specific place and time in history, but as a set of parameters in a model of natural selection. The values of those parameters represent a weighted sum of the environments experienced by the organism over the period of time relevant to explaining the currently observed value of the phenotype. For example, in studying the evolution of adaptations to high altitude, researchers seek to establish historical occupation patterns across the diverse landscapes that have been inhabited by populations that exhibit these adaptations (Beall [2014]). The ability to determine the parameters of the EEA is an important constraint on the rigorous use of evolutionary mismatch explanations. One relatively unproblematic application of the concept is when parameters that have been more or less constant through human evolution have changed due to documented recent developments, such as the virtual elimination of multicellular gut parasites in the developed world.

The EEA concept has also been criticised from the perspective of niche-construction theory. Nicheconstruction replaces the idea that populations evolve in response to the environment with a reciprocal relationship in which evolving populations also shape the environment. But niche-construction does not do away with the idea of adaptation. It couples the equations of change for genotypes (representing adaptation) to the equations of change for environmental variables (representing niche-construction) (Lewontin [1983], p. 282). An evolving population responds to selection pressures which themselves change as a function of the response to selection of the population. This approach to adaptation has recently been explored by Tanaka, Godfrey-Smith and Kerr ([2020]). The EEA in the traditional picture of evolution, ignoring niche construction, is a weighted average of the historical environments to which an evolving population has been exposed. It attempts to represent the spatial and temporal variation in 
historic environments in a tractable model. Niche construction complicates this picture because the different 'patches' of the environment are temporally ordered and the order in which they occur is a function of the changing composition of the population. But 'more complicated' does not necessarily mean 'intractable' (Tanaka et al. [2020]). If it is possible to successfully model evolutionary change whilst taking account of niche construction, then it will also be possible to model situations in which population mean fitness is reduced because organisms are unable to adapt to changes in the environment, including those induced by their own niche-constructing activities (the 'negative' niche construction of Odling Smee et al ([2003])).

Niche-construction theory is thus perfectly compatible with mismatch theory. The most sophisticated application of niche-construction theory to mismatch to date, that of Laland and Brown ([2006]), proposes the empirical hypothesis that niche construction in humans reduces adaptive lag or mismatch (they treat these as synonyms). They do not reject the idea of mismatch, and indeed they think it a "truism" that mismatch occurs widely ([2006], p. 98).

As we will see in Sections 2.2 and 2.3, when mismatch is identified on shorter timescales the reference environment in evolutionary medicine is not always the EEA. But the reference environment and the actual environment are always related by some kind of change. Fitness is reduced in the actual environment because the change in environment has exceeded the capacity of the organism to adapt. This difference between the scale of environmental change and the rate at which the organism can adapt to that change - whether by physiological, developmental or evolutionary means - is the theoretical core of the idea of mismatch in evolutionary medicine. It is this feature that allows mismatch to explain observed maladapted phenotypes: the organism is maladapted because the environment it occupies is not the environment to which it is adapted and it has been unable to catch up.

\subsection{Expected fitness and realised fitness}

Mismatch is used to explain maladaptation, not merely bad luck. The general mismatch schema (Figure 3) could be applied to the famous hypothetical of two identical organisms one of which is struck by a falling tree (Scriven [1959]). The squashed organism has lower fitness in its actual environment than it would have in the reference environment - the environment of the identical organism that does not get squashed. But no-one would seriously call this a 'mismatch' because there is no systematic relationship between the organism's phenotype and this piece of bad luck.

In this frivolous example, the utility function used to detect mismatch is 'realised fitness', the actual reproductive output of an organism, rather than 'expected fitness', the reproductive output an organism would have on average across the range of environmental conditions experienced by the population to which it belongs, weighted by the frequencies of those conditions (Charles H Pence and Grant Ramsey [2013]). This distinction between realized and expected fitness is at the heart of the propensity interpretation of fitness (Mills and Beatty [1979]; Charles H Pence and Grant Ramsey [2013]; Doulcier et al. [2021]), and the divergence between realized and expected fitness can be used to measure the amount of drift in a population (Grant Ramsey [2013]; Bourrat [2017]). The utility function relevant for natural selection and adaptation, and thus for evolutionary mismatch, is expected fitness, not realised fitness.

In evolutionary mismatch, the relevant measure is expected fitness, but the idea of mismatch has also been applied on shorter timescales, and this sometimes requires using realised fitness as a measure. In developmental mismatch, a phenotypically plastic organism makes an incorrect prediction ${ }^{8}$ about its future environment and in consequence develops a maladaptive phenotype. Detailed examples are given

\footnotetext{
${ }^{8}$ An anonymous referee has pointed out that 'prediction' might appear inappropriately mentalistic in this context. However, it is universally used in the literature on developmental and phenotypic plasticity and this façon de parler does not imply that biological mechanisms possess intentional states. For a recent philosophical investigation of the heuristic value (and pitfalls) of intentional language in evolutionary biology, see Okasha ([2018]).
} 
in Section 3, but for now, consider a water flea that grows defensive armour in response to chemical cues of the presence of predators, and then finds itself in a pool with no predators. The realised fitness of the flea would have been higher had it saved these resources and invested them in reproduction. ${ }^{9}$ In general, phenotypic plasticity can go wrong because the EEA is 'normal but noisy'. Most developmental decisions must be made with less than perfect information, so even an organism following an optimal decision rule will make errors at a predictable rate (as in the example of the water flea). An individual organism can thus maximise its expected fitness by following the optimal rule but fail to maximise its realised fitness because the optimal rule has a rate of error. Matthewson and Griffiths ([2017]) have argued that the inevitability of errors in mechanisms of 'predictive adaptive responses' (PAR) is a major original insight of evolutionary medicine. Organisms will sometimes be mismatched even in their EEA because phenotypic plasticity uses cues to predict which of a range of (normal) environments the organism will encounter in later life and these predictions are less than perfect (Peter D Gluckman and Hanson [2004]).

One very influential idea in evolutionary medicine is that an evolutionary mismatch can cause widespread developmental mismatch. A change in the environment can disrupt the relationship between cues earlier in life and environments later in life. The mechanism of plasticity that relies on that cue is evolutionary mismatched to the new environment. This leading to many individuals in the population being developmentally mismatched to their local environment. In such cases, organisms fail to maximise their expected fitness, as well as their realised fitness, because the cue-consequence relation in the actual environment is different from that in the EEA.

The scientific literature does not have distinct names for the two different scenarios that can account for an observed developmental mismatch, although the two alternative scenarios are clearly distinguished and the point that even under ideal circumstances development involves committing to a phenotype with less than perfect information is also made very clear (e.g. Peter D Gluckman and Hanson [2004], p. 1735; Kuzawa [2008], pp. 344-5). We now introduce some terminology to distinguish various mismatch scenarios (Figure 4 and Table 1).

\subsection{A taxonomy of mismatch phenomena}

We call a mismatch that occurs on the evolutionary timescale and to a constitutive phenotype, a 'simple evolutionary mismatch'. These mismatches reduce expected fitness, which often but not always means that realised fitness will be reduced for many individuals. The thrifty genotype hypothesis is an example of simple evolutionary mismatch.

When a mismatch occurs on the developmental timescale because natural selection has failed to keep pace with the changing environment we call this an 'evolutionary developmental mismatch'. These are cases where natural selection has not adapted the mechanism of plasticity to the changing environment. This failure of adaptation on an evolutionary timescale explains the failures of adaptation on the developmental timescale. Here again, both expected and realised fitness are reduced.

It has been suggested that the thrifty phenotype hypothesis is an example of evolutionary developmental mismatch, with modern environments systematically giving the developing fetus the wrong cue about its future environment. But researchers have emphasised that even in normal environments this mechanism may produce a thrifty phenotype which find itself living in nutritional abundance. This would eb example of our next category of mismatch:

\footnotetext{
${ }^{9}$ To be precise, the realised fitness of a flea which follows the correct decision rule in choosing its phenotype, and thus maximises its expected fitness, but which actually finds itself in the wrong environment, will be lower than the expected fitness unless other unsystematic factors - one or more lucky accidents - lead to the individual having a reproductive output that is higher than we would expect for a flea with this phenotype in that environment.
} 
When a mismatch occurs on the developmental timescale simply because a mechanism of phenotypic plasticity has produced the wrong phenotype for the actual environment we call this a 'simple developmental mismatch'. Such failures occur because even a perfectly adapted mechanism for making decisions under uncertainty must make some errors. No evolutionary mismatch is needed. These mismatches reduce the realised fitness of individuals, but not their expected fitness since, ex hypothesi, the decision rule is well-adapted to the EEA.

We make the same distinction between simple and evolutionary mismatch for mismatch phenomena occurring on the physiological timescales (Figure 2 and Table 1) and we discuss some examples of each in Section 3.

It is important to note that while we distinguish three timescales, physiological, developmental and evolutionary, this division is not rigid. Kuzawa ([2008]) adds a fourth, 'intergenerational' timescale (Figure 1) for mismatches generated by mechanisms of inter-generational adaptive plasticity ('parental effects' (Badyaev and Uller [2009])). In contrast, Raubenheimer, Simpson and Tait ([2012]) treat epigenetic and genetic mechanisms as operating on a single 'transgenerational' timescale. The important point is that mismatch can occur as a result of many different mechanisms, each of which acts on a specific timescale. Finer or coarser-grained timescales can be used to study particular aspects of mismatch.

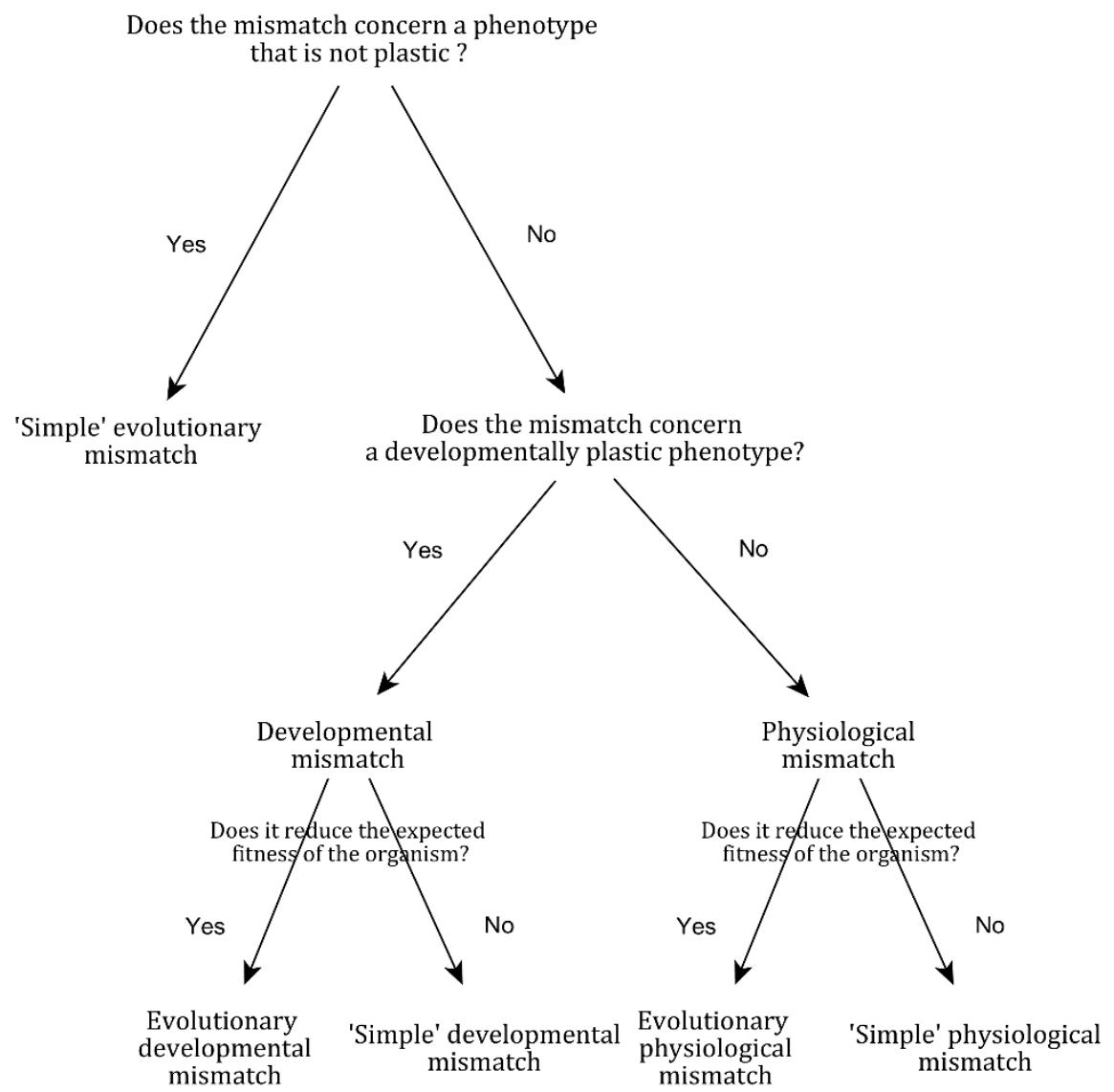

Figure 4. A taxonomy of mismatch phenomena. See Table 1 and text for explanation and examples. For physiological mismatch see Section 3 for examples. 


\begin{tabular}{|ll|}
\hline Mismatch & $\begin{array}{l}\text { Situation in which the fitness (expected or realised) } \\
\text { of a biological unit is decreased as a result of an } \\
\text { environmental change that exceeds the capacity of } \\
\text { the biological unit for immediate adaptation }\end{array}$ \\
\hline Simple Evolutionary Mismatch & $\begin{array}{l}\text { Mismatch in which the utility function is expected } \\
\text { fitness and the reference environment is the } \\
\text { Environment of Evolutionary Adaptedness (EEA). }\end{array}$ \\
& $\begin{array}{l}\text { A form of evolutionary mismatch which has } \\
\text { attracted particular attention in evolutionary } \\
\text { medicine Environmental change disrupts the } \\
\text { relationship between cue and consequence }\end{array}$ \\
implicitly assumed by a mechanism of phenotypic \\
plasticity, leading that mechanism to produce \\
maladapted phenotypes. Both expected and realised \\
fitness are reduced. \\
Mismatch in which the utility function is realised \\
fitness (but see fn.9) and the mechanism \\
responsible for the mismatch is phenotypic \\
plasticity. Simple Developmental Mismatch will \\
occur regularly in the EEA because developmental \\
decisions are made with less than perfect \\
information. \\
The phenomenon, included in the definitions of \\
mismatch by Kuzawa ([2008]), Gluckman et al \\
([2009]) and Raubenheimer, Simpson and Tait \\
([2012]), in which environmental change has \\
disrupted the relationship between cue and \\
consequence assumed by physiological \\
mechanisms of homeostasis or allostasis, so that \\
these mechanisms consistently generate phenotypic \\
changes which do not match the actual \\
environment.
\end{tabular}

Table 1. The theory of mismatch in contemporary evolutionary medicine. Note that mismatch on the physiological and developmental timescales requires phenotypic plasticity. Simple evolutionary mismatch does not and can apply to constitutive phenotypes. 


\section{A case-study: mismatch in nutritional ecology}

In this section, we examine the use of mismatch in nutritional ecology, a field of evolutionary medicine which takes a comparative and evolutionary perspective on obesity and other aspects of metabolic syndrome (see 'Principles of Evolutionary Medicine' (1st Ed.) Peter D. Gluckman et al. [2009], Box 8.5).

Some leading figures in nutritional ecology define mismatch as follows:

"Mismatch occurs when the timescale and/or magnitude of environmental change exceeds the combined capacity of adaptation owing to homeostatic mechanisms, phenotypic plasticity and transgenerational adaptation." (Raubenheimer et al. [2012], p. 1641)

To begin with we will examine evolutionary mismatch in nutritional ecology, before explaining how the framework encompasses developmental and physiological mismatch. Evolutionary mismatch occurs when the organism's expected fitness in its actual environment is significantly lower than in the EEA.

The environment in nutritional ecology consists of 'foods' available to the animal. These can be combined into 'meals' in proportions dictated by the animal's feeding behaviour. The overall combination of foods an animal consumes over some period is its 'diet'. The phenotype in nutritional ecology is the regulatory mechanisms that determine which diet an animal will consume in a given food environment. Adaptation is defined in terms of whether a diet maximises fitness. The fitnessmaximising diets can be represented as a 'nutritional intake target', a point in a multi-dimensional space whose axes represent macronutrients - fat, carbohydrate and protein - and micronutrients - minerals such as iodine and vitamins such as ascorbic acid. (Machovsky-Capuska et al. [2016] and see Figure 5)

An animal's ability to reach its nutritional intake target is constrained by the food environment. If no individual food contains the correct proportions of macro- and micronutrients, then animals try to consume the available foods in such proportions that their overall diet contains the correct proportions of macro- and micronutrients (Simpson and Raubenheimer [2012]). Whether this is possible depends on the content of the available food items. For example, an animal that feeds on fruit and leaves may find it impossible to reach its intake target in the off-season when trees are not fruiting. In such cases, although 'food' is abundant it consists entirely of one food, leaves, which contain a higher proportion of protein than the optimal diet (Cui et al. [2018]).

If the environment makes it impossible to reach the intake target, then an animal must follow a 'rule of compromise' (Simpson and Raubenheimer [2012]) which determines which nutrients it will overconsume and which it will underconsume. For example, many animals, including humans, prioritise protein over other macronutrients. They defend a protein intake target by over or under consuming fats and carbohydrates (Gosby et al. [2011]). Many other 'rules of compromise' are possible, such as an 'equal distance' rule which balances the size of the deficits and surpluses in alternative macronutrient groups and which may characterise the behaviour of some generalist primates (Cui et al. [2018]). The 'rule of compromise' is itself an adaptation which minimises the fitness cost to the animal of finding itself in an environment in which it cannot reach its nutritional intake target. Seasonal fluctuation in the availability of different foods provides an obvious environment for the evolution of an adaptive rule of compromise. In a novel, sub-optimal food environment, however, all bets are off and the diet generated by an evolved rule of compromise may be highly maladaptive (Figure 5). 


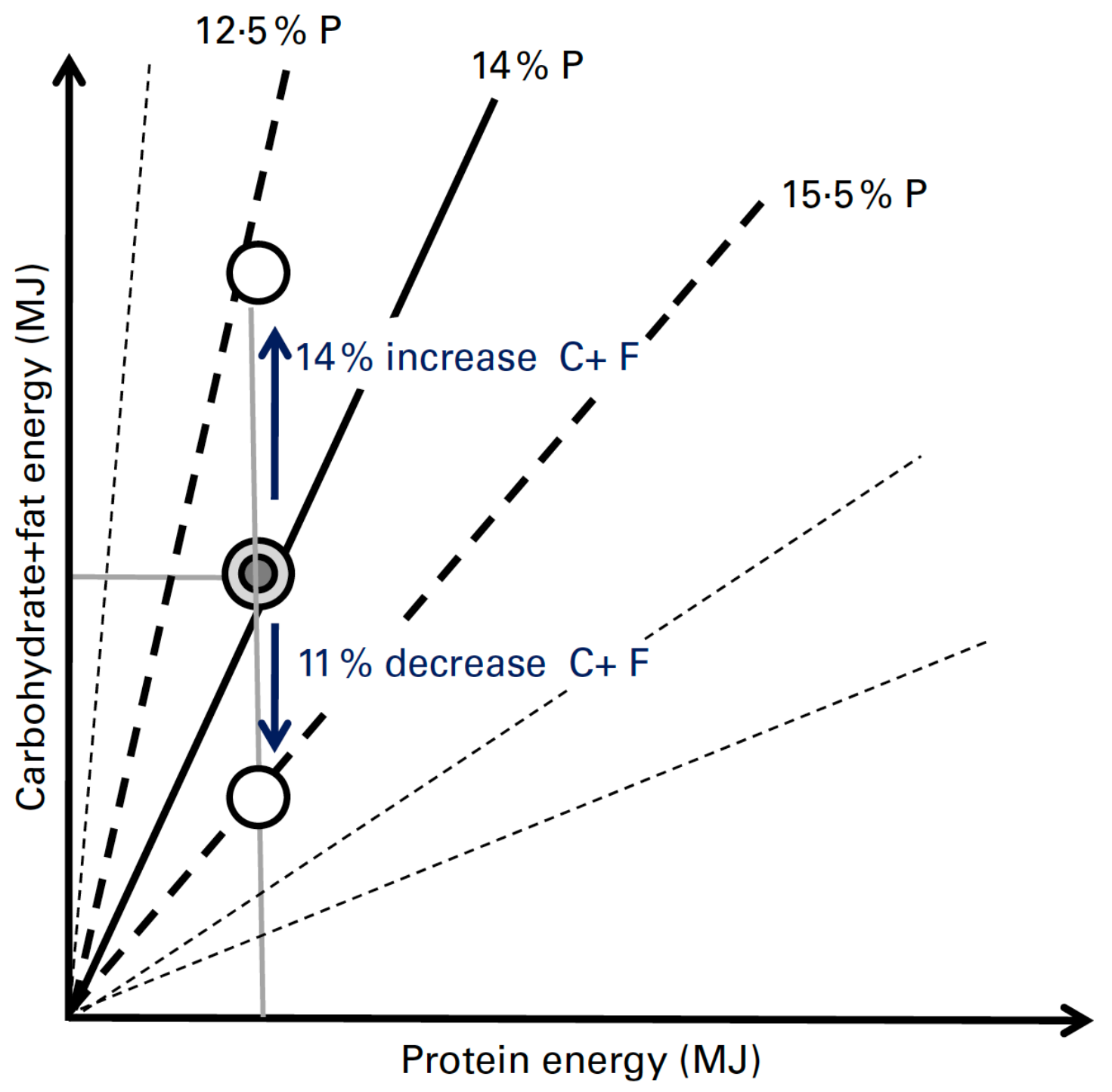

Figure 5. Hypothetical example to illustrate a 'rule of compromise'. In this case, the rule is 'strict protein prioritisation' where an absolute protein intake target dominates the choice of diet. Under this rule, a small change in the ratio of protein to other macronutrients in the possible diets available to the animal can lead to a large change in consumption of other nutrients. In this example, "a $1.5 \%$ decrease in the proportion of energy from protein (from 14 to $12.5 \%$ ) will result in a $14 \%$ increase in the amount of carbohydrate and fat eaten. Conversely, a $1.5 \%$ decrease in dietary protein density will correspond with a $11 \%$ decrease in non-protein energy eaten." (from Raubenheimer et al. [2015], S29)

The 'protein leverage' effect shown in Figure 5 is a proposed explanation of the increase in obesity in humans and their companion animals in recent decades (Raubenheimer et al. [2015]). This is a classic example of an evolutionary mismatch hypothesis. In the EEA foods high in fat and carbohydrate but low in protein are rare. Protein prioritisation is adaptive on the rare occasions when protein is rare, because of the physiological challenge of storing protein. But foods high in fat and carbohydrate and low in protein are now ubiquitous. As a result, the evolved rule of compromise causes humans and other animals to consistently consume more calories than they can use or store without becoming obese. This widespread pattern of maladaptive behaviour is explained by the inability of natural selection to change 
the nutritional phenotype quickly enough to keep pace with the changing nutritional environment. The rule of compromise minimised the loss of fitness in hostile patches of the EEA. In current environments, this hypothesis suggests, the rule leads to obesity, diabetes, heart disease and other elements of metabolic syndrome. ${ }^{10}$

\section{How mismatch integrates development and evolution}

The mismatch between the rule of compromise and a novel environment just described is a simple evolutionary mismatch. In simple evolutionary mismatch, the organism has a constitutive phenotype ${ }^{11}$ which is maladapted in the new environment.

Another adaptation which may undergo simple evolutionary mismatch is the physiological needs of the organism which the nutritional intake target is designed to satisfy. An organism whose physiological needs cannot be met by a novel environment is mismatched to that environment. But the physiological needs of an organism co-evolve with its dietary environment. There is evidence that humans with different biogeographic origins differ in the extent to which specific levels of obesity are risk-factors for Type-II diabetes (Chiu et al. [2011]). If correct, this would suggest that these populations are physiologically adapted to slightly different food environments, just as Neel speculated ([1958]). As a result, some human populations may experience a greater degree of mismatch to modern, industrialised food-environments than others (but see fn. 11)

One way in which evolution and development interact is that evolutionary mismatch can cause developmental mismatch. In evolutionary developmental mismatch, a mechanism of developmental plasticity produces a mismatched phenotype because the cue-consequence relationship implicitly assumed by the mechanism does not hold in the new environment. For example, the mechanisms by which infants use cues in milk to form taste preferences are mismatched to the environment of formula feeding. Avoiding tastes not found in infant formula, for example the tastes of vegetables (Domínguez [2021]), is maladaptive. Evolutionary developmental mismatch can have very dramatic consequences: apparently insignificant changes to the environment may have dramatic consequence for population health if organisms use them as cues to determine their developmental trajectory. This insight is at the heart of DOHaD research on 'predictive adaptive responses' (Peter D. Gluckman et al. [2005]; Gluckman et al. [2007]).

But developmental mismatch is not just a special case of evolutionary mismatch. It is an independently defined phenomenon that only sometimes results from evolutionary mismatch. Simple evolutionary mismatch has potentially very different implications for medicine. As we explained in Section 2, developmental decisions are almost always decisions under uncertainty so that even an optimal decision rule generates errors. Gluckman and collaborators argued that a predictive adaptive response may produce "a continuous range of human metabolic "morphs" representing a suite of integrated responses to the environmental cues received in utero or by the neonate which establish the setpoints of the metabolic and related systems." (Gluckman et al. [2007], p. 14). As a result, "even when fetal growth falls within the normal range, being born into an enriched postnatal environment can create a mismatch". (Peter D Gluckman and Hanson [2004], p. 1735). So a PAR will make errors, not because it is operating in a novel environment, but because the best possible decision rule still has a rate of error. These errors are simple developmental mismatches.

\footnotetext{
${ }^{10}$ An anonymous referee has asked us to increase the focus on human obesity in our discussion. But we cannot do justice to complex and unresolved empirical issues around human obesity in the space available here and these remarks should only be taken as hypothetical illustrations of the principle of nutritional ecology. An accessible attempt to explain the potential realworld applications of these principles is Eat Like the Animals (Raubenheimer and Simpson [2020])

${ }^{11}$ In biology a 'constitutive' phenotype is one that is not plastic in the sense defined in $\mathrm{fn}$. 3 . Whether rules of compromise and nutritional intake targets are, in fact, plastic in some species is a complex, empirical issue.
} 
The same approach can be applied mutatis mutandis to distinguish 'evolutionary physiological mismatch' from 'simple physiological mismatch'. Such cases are normally descried simply as failures of homeostasis or allostasis. When the actual environment differs from that predicted by the operation of the mechanism, the physiological state of the organism is mismatched to the actual environment. For example, an organism that has elevated cortisol levels in response to cues of a hostile environment but is actually in a benign environment is mismatched to that environment. This organism will pay the short-term physiological cost of increasing cortisol level on realised fitness when this is unnecessary.

The theory of mismatch draws attention to the parallels between physiological mismatch and mismatch on larger timescales. Suppose that some failure of homeostasis or allostasis occurs strikingly often in a population, and hence calls for an explanation. One possible explanation is a downstream consequence of evolutionary mismatch or 'evolutionary physiological mismatch'. This would involve an evolutionary mismatch between the physiological mechanism and a novel environment because cueconsequence relationship implicit in the design of a physiological mechanism no longer holds in that environment. The other possible explanation parallels 'simple developmental mismatch'. In 'simple physiological mismatch' a homeostatic mechanism follows a rule which maximises expected fitness, but because the environment is noisy even the optimal rule often fails to maximise realised fitness (see Figure 4). The 'smoke detector principle' in evolutionary medicine is one example (Nesse [2005]).

There is nothing specific to nutrition about this need to recognise mismatch phenomena on multiple timescales. For example, in the study of adaptation to altitude: "Knowing how long the population has resided at altitude is important for considering the potential for modes of adaptation that occur on time scales ranging from short (reversible acclimatization), to intermediate (developmental) and long (genetic)." (Beall [2014], p. 253). The different ways in which different human populations have adapted to living at high altitude produce the same range of mismatch phenomena that we have attempted to illustrate using nutrition and their health impacts are the topic of current research in evolutionary medicine (S. Corbett, pers. comm).

The theory of mismatch integrates all these phenomena into a theory of how physiology, development and evolution interact with each other and with environmental change to explain health outcomes. As an example of the power of this integrative approach, consider how it improves our understanding of homeostasis. Once we see homeostasis as a mechanism of fitness-tracking on short timescales we can analyse it in the framework of life-history theory, the same framework we use to understand the evolution of constitutive phenotypes and of developmental plasticity. It is ultimately inadequate to regard homeostasis (and its relatives homeorhesis, allostasis, etc) as targeting anything other than the maximisation of fitness (Raubenheimer et al. [2012]). The values and ranges of internal variables that are the targets for these physiological mechanisms are not optimal in any simple, physiological sense, but rather represent trade-offs between multiple goals where the implicit relative fitness payoffs of those goals are those from the EEA.

From this perspective the traditional idea that nutrition science should identify an 'optimal diet' appears naïve. There is no single 'optimal diet' since different macronutrient ratios are 'optimal' for maximum lifespan, maximum lifetime reproductive output, immune functioning, etc (Cotter et al. [2011]; for an overview, see Raubenheimer et al. [2012], pp. 1637-9; Solon-Biet et al. [2015]). The prediction is that the observed nutritional intake target, which is itself a complex plastic trait that changes over the lifecourse, will represent a trade-off between these different goals that reflects their relative importance in the EEA.

Conversely, the theory of mismatch integrates physiological and developmental understanding into evolution. An organism with a mechanism of physiological or developmental plasticity that allows to adapt to some range of environmental change does not need to undergo adaptation by natural selection in that range of environments. Thus, in order to predict the response to selection biologists need to take into account the capacity of an organism for physiological and developmental adaptation. 
Physiological plasticity, intra- and inter-generational developmental plasticity, and evolution by natural selection are all means to the same end - tracking changing environments so as to maximise fitness. The design of each of these mechanisms reflects the same evolutionary compromises between multiple life-history goals. These mechanisms interact with one another, forming a suite of means by which organisms can cope with environmental heterogeneity. It is this focus on adaptive tracking, and on multiple mechanisms and modes of adaptation, that we take to be the distinctive core of research into mismatch in evolutionary medicine.

\section{Comparison to Previous Accounts of Mismatch}

Relatively little philosophical work has been done on the concept of mismatch. However, two of the three available accounts explicitly claim to characterise how the term 'mismatch' is used in evolutionary medicine and both contradict the account we have given. This clearly merits some discussion.

\subsection{Lloyd, Wilson and Sober 2011}

The aim of Lloyd et al's paper is to argue that evolutionary mismatch explanations are testable hypotheses and not mere 'just so-stories'. They share the definition of evolutionary mismatch found in evolutionary medicine textbooks and advocated above:

"An evolutionary mismatch can be defined as a negative consequence that results from a trait that evolved in one environment being placed in another environment." (Lloyd et al. [2011], p. 4, emphasis in original).

Our disagreements with Lloyd et al are minor. They do not explicitly distinguish evolutionary mismatch from developmental mismatch, but they do recognize that "cases of mismatch involving phenotypic plasticity" are of particular interest ([2011], p. 14). They introduce predictive adaptive responses (PARs) as an example of evolutionary mismatch involving phenotypic plasticity. But although their source for PARs draws attention to simple developmental mismatch - "even when fetal growth falls within the normal range, being born into an enriched postnatal environment can create a mismatch." (Peter D Gluckman and Hanson [2004], p. 1735) - Lloyd et al do not seem to recognise this. They recognise only two ways in which a PAR can produce a maladapted phenotype, either environmental change has divorced cue and consequence or there has been "a disruption of normal development" (Lloyd et al. [2011], p. 15). But this is not an exhaustive dichotomy. Developmental mismatch can occur independently of evolutionary mismatch and without any disruption of normal development. Gluckman gave one example. Another is that any plastic response to a normal but noisy environment will have a predictable error rate, which will explain part of the burden of disease (Matthewson and Griffiths [2017]). So many individual differences in health outcomes will result from simple developmental mismatch.

\subsection{Morris 2018}

Like Lloyd et al, Morris focuses on evolutionary mismatch. The distinctive feature of his proposed definition is that mismatch is assessed by comparing the actual environment to an 'optimal' environment:

"CC: evolutionary mismatch obtains when an organism $\mathrm{O}$ is in an actual environment $\mathrm{E}_{\mathrm{a}}$ such that $\mathrm{O}$ 's fitness is lower than it would be in an optimal environment $\mathrm{E}_{\mathrm{mf}} . "$ (Morris [2018])

We do not think that using an optimal environment rather than the EEA as the reference environment reflects how the concept is used in evolutionary medicine. Following Levins ([1968]), Morris states that "the optimal environment is the environment in which the organism's fitness is maximized: in other 
words, the optimal environment is that in which the organism's fitness is as high as it can possibly be." (Morris [2018], p. 1) An immediate problem is that this will classify almost all actual organisms as mismatched, because almost no organism lives in an optimal environment. For example, one limiting factor for plants is nitrogen. Most non-leguminous plants are not in an optimal environment with regards to nitrogen. This is why nitrogen-based fertilizers are so widely used. If we follow Morris, the world's rainforests are all spectacularly mismatched to the nitrogen-impoverished soils with which they have co-evolved.

Morris is well aware of this problem, part of what he calls the "overbroadness objection" (Morris [2018], p. 17). He offers a thoughtful defense of what he recognises will seem an unintuitive consequence. He notes that although the environment can typically be improved for any organism, a ceiling point will be obtained for any given variable, so optimal environments will probably not be impossible utopias that go beyond anything of scientific interest. However, as he recognises, that ceiling point will usually be far above the value in actual environments: life is harsh.

Another disadvantage of Morris's definition is that mismatch so defined is no longer an explanatory concept. Mismatch explanations depend on using the EEA as the reference environment - the observed phenotype is explained by actual, historical adaptation to the EEA and the failure of the organism to match the actual environment is explained by the difference between the scale of environmental change and the rate of adaptation. Morris's proposal to use the optimal environment as the reference environment identifies 'mismatch' with 'maladaptation' and renders any explanation of maladaptation using the idea of mismatch circular - we observe maladaptation because the environment is not optimal for the organism. Another way to put this point is that whereas we think evolutionary mismatch is adaptive lag, as we explained in Section 1, Morris thinks mismatch is the phenomenon (maladaptation) that is sometimes explained by adaptive lag. That would be a coherent way to use the term 'mismatch', but we don't think it is how the term is actually used. Mismatch is one just one possible explanation of maladaptation. That is why evolutionary medicine textbooks list mismatch alongside other explanations of maladaptation such as constraints on evolution or accidents of demographic history (e.g. Stearns and Medzhitov [2015]; Peter D Gluckman et al. [2016]).

We do not disagree when Morris argues there is a coherent way in which we can think about evolutionary processes using his definition of mismatch. But we think Morris's proposed definition of 'mismatch' would be a less useful theoretical construct than that we actually see used in evolutionary medicine research today.

\subsection{Cofnas 2016}

The analysis of the idea of mismatch most at odds with ours is Cofnas, who "defines mismatch as deviations in the environment that render biological traits unable, or impaired in their ability, to produce their selected effects" (Cofnas [2016], p. 507). By 'selected effects' Cofnas means the effects for which the trait is an adaptation. This definition leads him to reject Lloyd et al's ([2011]) view that mismatch is restricted to environmental changes that have a deleterious effect. Cofnas uses the terms "mismatch" and "novelty" as synonyms ([2016], pp. 509, 522) and then argues against Lloyd et al as follows:

"Evolutionary novelty is defined as deviations in the environment that prevent biological traits from performing, or impair their ability to perform, such functions. Such impairment is not necessarily bad for organisms from the standpoint of either fitness or welfare. Mice may possess mechanisms the proper function of which is to escape from cats. These mechanisms do not perform these functions in cat-free environments, but cat-free environments are good for mice." (Cofnas [2016], pp. 510-1)

Cofnas claims that, "scientists have generally been working without a clear definition of mismatch." ([2016], pp. 507-8) but this is not correct, as we have seen above. Take the definition we have used several times above: "Mismatch occurs when the timescale and/or magnitude of environmental change 
exceeds the combined capacity of adaptation owing to homeostatic mechanisms, phenotypic plasticity and transgenerational adaptation." (Raubenheimer et al. [2012], p. 1641) Not only is this a clear definition, it can be used to clarify Cofnas' thought-experiment. A mouse in a house with no cats has certainly not exceeded its capacity to adapt to the environment. 1. Physiological adaptation: it adapts by not being frightened and thus not paying the physiological and opportunity costs associated with fear and anxiety. 2. Developmental adaptation: mice in secure environments develop with lowered basal cortisol levels and heightened exploratory behaviour, although this may take two generations to kick in via an epigenetically mediated parental effect (Meaney [2001]). 3. Evolutionary adaptation: mice have no need to adapt by natural selection because at a population level the mouse phenotype is not mismatched. Mice live in a patchy environment of cat-free and cat-filled houses and successfully track that environment using physiology and developmental plasticity. In this respect, mice live in their EEA.

When we apply this definition from actual evolutionary medicine research to Cofnas's example we get some interesting insights about mouse biology. Conversely, if we apply Cofnas's definition to the actual scientific models outlined in Section 3 we get the result that every animal in a novel food environment where it can reach its nutritional intake target is mismatched because the animal's rule of compromise does not perform its adaptive function. An animal feeding on a single novel food which constitutes an optimal diet is even more mismatched, according to Cofnas, since none of its adaptations for reaching its nutritional intake target perform their adaptive functions in that environment. We cannot see what value this definition could have for biomedical research and that is perhaps why nothing like this appears in evolutionary medicine.

Cofnas's definition of mismatch is plainly inconsistent with the definitions quoted in Section 1 from evolutionary medicine textbooks (Peter D. Gluckman et al. [2009]; Stearns and Medzhitov [2015]; Peter D Gluckman et al. [2016]) and influential theoretical papers (Bateson et al. [2004]; P. D. Gluckman et al. [2009]). Cofnas does not discuss these, or any other definitions of mismatch from the scientific literature, so we are unclear what his objections to them are. However, every definition we have encountered sides with Lloyd et al over Cofnas on the issue of whether mismatch reduces fitness.

Cofnas claims that his analysis applies to evolutionary medicine but his main concern is evolutionary psychology and in particular research on the evolution of general intelligence (' $\mathrm{g}$ '). But what is relevant here is that his analysis does not correctly characterise how mismatch is understood in evolutionary medicine, either explicitly in published definitions or implicitly in models and experiments like those described above.

\section{Conclusion}

We can speak in broad terms of a 'mismatch' with the environment whenever a gene, organism or other biological unit performs better on some measure in some reference environment than in its actual environment (Figure 3). In evolutionary medicine, however, mismatch refers to cases where the scale of environmental change has exceeded the capacity of organisms to adapt. The discrepancy between the scale of environmental change and the capacity for adaptation is the theoretical core of the idea of mismatch in evolutionary medicine. It is this feature that allows mismatch to explain observed maladaptation.

The theory of mismatch found in contemporary evolutionary medicine merges two ideas that were both labelled with the term 'mismatch' some time after they were recognised and discussed in the scientific literature. The first idea is 'evolutionary mismatch' (Riggs [1993]): adaptation by natural selection sometimes fails to keep pace with environmental change. This idea emerged in the modern synthesis, where it was known as 'adaptive lag'. The second idea is 'developmental mismatch' (Kuzawa [2008]): mechanisms of developmental and phenotypic plasticity produce maladaptive phenotypes when an environmental cue early in development does not correspond to the environment actually experienced later in the lifecourse. These developmental mismatches are sometimes (but not always) the result of an evolutionary mismatch between the mechanism of plasticity and the modern environment. This 
second idea originated in the field of Developmental Origins of Health and Disease (DOHaD). The first idea was first labelled 'mismatch' in the late ' 80 s and the second was first labelled 'mismatch' in the late '90s (see Section 1 and Supplementary materials). In the 2000s there was a deliberate effort to synthesise these two research traditions, with interdisciplinary workshops and multi-author 'manifesto' articles in prestigious journals.

In contemporary evolutionary medicine the idea of mismatch frames the study of how organisms track changing environments on multiple scales so as to maximise fitness, and of where this goes wrong.

The resultant body of theory recognises that evolutionary and developmental and physiological mismatch interact in numerous ways, and hence that the study of mismatch must integrate evolutionary and developmental and physiological studies. As just noted, evolutionary mismatch may cause widespread developmental or physiological mismatch. Conversely, the existence of mechanisms of physiological and developmental plasticity 'buffer' genotypes against environmental change. Mismatch is a rich example of how evolutionary, and evolutionary-developmental, reasoning can contribute to biological and medical science.

\section{References}

Badyaev, A. V. and Uller, T. [2009]: 'Parental Effects in Ecology and Evolution: Mechanisms, Processes, and Implications', Philosophical Transactions of the Royal Society, Biological Sciences, 364, pp. 1169-77.

Bateson, P. [1997]: 'Design for a Life', in D. Magnusson (ed.), The Lifespan Development of Individuals: Behavioral, Neurobiological, and Psychosocial Perspectives: A Synthesis, Cambridge University Press.

[2001]: 'Fetal Experience and Good Adult Design', International Journal Of Epidemiology, 30, pp. 928-34.

Bateson, P., Barker, D., Clutton-Brock, T., Deb, D., D’Udine, B., Foley, R. A., Gluckman, P., Godfrey, K., Kirkwood, T., Lahr, M. M., McNamara, J., Metcalfe, N. B., Monaghan, P., Spencer, H. G. and Sultan, S. E. [2004]: 'Developmental Plasticity and Human Health', Nature, 430, pp. 41921.

Bateson, P. P. G. and Martin, P. [1999]: 'Design for a Life: How Behavior and Personality Develop', London: Jonathan Cape.

Beall, C. M. [2014]: 'Adaptation to High Altitude: Phenotypes and Genotypes', Annual Review of Anthropology, 43, pp. 251-72.

Bourrat, P. [2017]: 'Explaining Drift from a Deterministic Setting', Biological Theory, 12, pp. 27-38.

Bowlby, J. [1969]: 'Attachment and Loss', Vol. 1: Attachment London: The Hogarth Press and the Institute of Psycho-Analysis, $\quad<$ http://www.pepweb.org.ezproxy1.library.usyd.edu.au/document.php?id=IPL.079.0001A>.

Buller, D. J. [2005]: 'Adapting Minds: Evolutionary Psychology and the Persistent Quest for Human Nature', Cambridge, MA: MIT Press/Bradford Books.

Charles H Pence and Grant Ramsey [2013]: 'A New Foundation for the Propensity Interpretation of Fitness', The British Journal for the Philosophy of Science, 64, pp. 851-81. 
Chiu, M., Austin, P. C., Manuel, D. G., Shah, B. R. and Tu, J. V. [2011]: 'Deriving Ethnic-Specific BMI Cutoff Points for Assessing Diabetes Risk', Diabetes Care, 34, pp. 1741-8.

Cofnas, N. [2016]: 'A Teleofunctional Account of Evolutionary Mismatch', Biology \& Philosophy, 31, pp. 507-25.

Cotter, S. C., Simpson, S. J., Raubenheimer, D. and Wilson, K. [2011]: 'Macronutrient Balance Mediates Trade-Offs between Immune Function and Life History Traits', Functional Ecology, 25, pp. 186-98.

Cui, Z.-W., Wang, Z.-L., Shao, Q., Raubenheimer, D. and Lu, J.-Q. [2018]: 'Macronutrient Signature of Dietary Generalism in an Ecologically Diverse Primate in the Wild', Behavioral Ecology, 29, pp. 804-13.

Dobzhansky, T. [1963]: 'Cultural Direction of Human Evolution - A Summation', Human Biology, 35, pp. 311-6.

Domínguez, P. R. [2021]: 'A New Look at Early Exposure to the Flavors of the Available Vegetables as Foundational Mechanism of Vegetable Consumption Habits and Recipes of VegetablesBased Dishes', Critical Reviews in Food Science and Nutrition, 61, pp. 855-66.

Doulcier, G., Takacs, P. and Bourrat, P. [2021]: 'Taming Fitness: Organism-Environment Interdependencies Preclude Long-Term Fitness Forecasting', BioEssays, 43, p. 2000157.

Eaton, S. B., Konner, M. and Shostak, M. [1988]: 'Stone Agers in the Fast Lane: Chronic Degenerative Diseases in Evolutionary Perspective', The American Journal of Medicine, 84, pp. 739-49.

Gluckman, Peter D and Hanson, M. A. [2004]: 'Living with the Past: Evolution, Development, and Patterns of Disease', Science, 305, pp. 1733-6.

Gluckman, Peter D. and Hanson, M. A. [2004a]: 'The Developmental Origins of the Metabolic Syndrome', Trends in Endocrinology \& Metabolism, 15, pp. 183-7.

[2004b]: 'The Fetal Matrix: Evolution, Development and Disease', Cambridge, UK: Cambridge University Press.

Gluckman, Peter D, Hanson, M. A., Spencer, H. G. and Bateson, P. P. G. [2005]: 'Environmental Influences during Development and Their Later Consequences for Health and Disease: Implications for the Interpretation of Empirical Studies', Proc. R. Soc. B, 272, pp. 671-7.

Gluckman, Peter D., Hanson, M. A. and Spencer, H. G. [2005]: 'Predictive Adaptive Responses and Human Evolution', Trends in Ecology \& Evolution, 20, pp. 527-33.

Gluckman, P. D. and Hanson, M. A. [2006]: 'Mismatch: Why Our World No Longer Fits Our Bodies', Oxford University Press.

Gluckman, P. D., Hanson, M. A. and Beedle, A. S. [2007]: 'Early Life Events and Their Consequences for Later Disease: A Life History and Evolutionary Perspective', Am J Hum Biol, 19, pp. 1-19.

Gluckman, Peter D., Beedle, A. and Hanson, M. A. [2009]: 'Principles of Evolutionary Medicine', Oxford; New York: Oxford University Press.

Gluckman, P. D., Hanson, M. A., Bateson, P., Beedle, A. S., Law, C. M., Bhutta, Z., Anokhin, K., Bougnères, P., Chandak, G., Dasgupta, P., Smith, G., Ellison, P., Forrester, T., Gilbert, S., Jablonka, E., Kaplan, H., Prentice, A., Simpson, S., Uauy, R. and West-Eberhard, MJ. [2009]: 
'Towards a New Developmental Synthesis: Adaptive Developmental Plasticity and Human Disease', Lancet, 373, pp. 1654-7.

Gluckman, Peter D, Beedle, A. S., Buklijas, T., Low, F. and Hanson, M. A. [2016]: 'Principles of Evolutionary Medicine', 2 edition., Oxford, United Kingdom: Oxford University Press.

Gluckman, Peter D., Buklijas, T. and Hanson, M. A. [2016]: 'The Developmental Origins of Health and Disease (DOHaD) Concept', in The Epigenome and Developmental Origins of Health and Disease, Elsevier, pp. 1-15, $<$ https://linkinghub.elsevier.com/retrieve/pii/B9780128013830000013>.

Godfrey-Smith, P. [2001]: ‘Three Kinds of Adaptationism', in S. Orzack and E. Sober (eds), Optimality and Adaptation, Cambridge: Cambridge University Press, pp. 335-57.

Gosby, A. K., Conigrave, A. D., Lau, N. S., Iglesias, M. A., Hall, R. M., Jebb, S. A., Brand-Miller, J., Caterson, I. D., Raubenheimer, D. and Simpson, S. J. [2011]: 'Testing Protein Leverage in Lean Humans: A Randomised Controlled Experimental Study’, PLOS ONE, 6, p. e25929.

Grant Ramsey [2013]: ‘Driftability’, Synthese (Dordrecht), 190, pp. 3909-28.

Hales, C. N. and Barker, D. J. P. [1992]: 'Type 2 (Non-Insulin-Dependent) Diabetes Mellitus: The Thrifty Phenotype Hypothesis', Diabetologia, 35, pp. 595-601.

Kuzawa, C. W. [2008]: 'The Developmental Origins of Adult Health: Intergenerational Inertia in Adaptation and Disease', in Evolutionary Medicine and Health: New Perspectives, Oxford University Press, pp. 325-49.

Laland, K. N. and Brown, G. R. [2002]: 'Sense and Nonsense: Evolutionary Perspectives on Human Behaviour', Oxford University Press.

— [2006]: 'Niche Construction, Human Behavior, and the Adaptive-Lag Hypothesis', Evolutionary Anthropology: Issues, News, and Reviews, 15, pp. 95-104.

Levins, R. [1968]: 'Evolution in Changing Environments: Some Theoretical Explorations.', Princeton, NJ: Princeton University Press.

Lewontin, R. C. [1983]: 'Gene, Organism \& Environment', in D. S. Bendall (ed.), Evolution: From Molecules to Man, Cambridge: Cambridge University Press, pp. 273-85.

Lloyd, E., Wilson, D. S. and Sober, E. [2011]: 'Evolutionary Mismatch And What To Do About It: A Basic Tutorial', Unpublished Manuscript, p. 23 p. 23.

Machovsky-Capuska, G. E., Senior, A. M., Simpson, S. J. and Raubenheimer, D. [2016]: 'The Multidimensional Nutritional Niche', Trends in Ecology \& Evolution, 31, pp. 355-65.

Mandler, G. [1984]: 'Mind and Body: The Psychology of Emotion and Stress', New York: Norton.

Manus, M. B. [2018]: 'Evolutionary Mismatch', Evolution, Medicine, and Public Health, 2018, pp. $190-1$.

Matthewson, J. and Griffiths, P. E. [2017]: 'Biological Criteria of Disease: Four Ways of Going Wrong', The Journal of Medicine and Philosophy: A Forum for Bioethics and Philosophy of Medicine, 42, pp. 447-66.

Mayr, E. [1942]: 'Systematics and the Origin of Species from the Viewpoint of a Zoologist', Columbia University Press. 
Meaney, M. J. [2001]: 'Maternal Care, Gene Expression, and the Transmission of Individual Differences in Stress Reactivity across Generations.', Annual Review Neuroscience, 24, pp. 1161-92.

Mills, S. and Beatty, J. [1979]: 'The Propensity Interpretation of Fitness', Philosophy of Science, 46, pp. 263-86.

Morris, R. [2018]: 'Stranger in a Strange Land: An Optimal-Environments Account of Evolutionary Mismatch', Synthese, <https://doi.org/10.1007/s11229-018-01915-x>.

Neel, J. V. [1958]: 'The Study of Natural Selection in Primitive and Civilized Human Populations', Human Biology, 30, pp. 43-72.

[1962]: 'Diabetes Mellitus: A “Thrifty" Genotype Rendered Detrimental by "Progress"?', American Journal of Human Genetics, 14, pp. 353-62.

[1989]: 'Update to "The Study of Natural Selection in Primitive and Civilized Human Populations"”, Human Biology, 61, pp. 811-23.

Nesse, R. M. and Williams, G. C. [1994]: 'Why We Get Sick: The New Science of Darwinian Medicine', New York: Times Books.

Nesse, R. M. [2005]: 'Natural Selection and the Regulation of Defenses: A Signal Detection Analysis of the Smoke Detector Principle', Evolution and Human Behavior, 26, pp. 88-105.

Odling-Smee, F. J., Laland, K. N. and Feldman, M. W. [2003]: 'Niche Construction: The Neglected Process in Evolution', Princeton: University Press.

Ogburn, W. F. [1922]: 'Social Change with Respect to Culture and Original Nature', New York: B. W. Huebsch, Inc., <https://archive.org/details/socialchangewith00ogburich/page/n1>.

Okasha, S. [2018]: ‘Agents and Goals in Evolution', Oxford University Press.

'Physiological Adaptation - Definition and Examples - Biology Online Dictionary', [2019, October 7]: Biology Articles, Tutorials \& Dictionary Online, $<$ https://www.biologyonline.com/dictionary/physiological-adaptation>.

Raubenheimer, D., Simpson, S. J. and Tait, A. H. [2012]: 'Match and Mismatch: Conservation Physiology, Nutritional Ecology and the Timescales of Biological Adaptation', Phil. Trans. $R$. Soc. B, 367, pp. $1628-46$.

Raubenheimer, D., Machovsky-Capuska, G. E., Gosby, A. K. and Simpson, S. [2015]: 'Nutritional Ecology of Obesity: From Humans to Companion Animals', The British Journal of Nutrition, 113 Suppl, pp. S26-39.

Raubenheimer, D. and Simpson, S. J. [2020]: 'Eat Like the Animals: What Nature Teaches Us About Healthy Eating', William Collins.

Richardson, S. S. [2021]: 'The Maternal Imprint: The Contested Science of Maternal-Fetal Effects', University of Chicago Press.

Riggs, J. E. [1993]: 'Stone-Age Genes and Modern Lifestyle: Evolutionary Mismatch or Differential Survival Bias', Journal of Clinical Epidemiology, 46, pp. 1289-91. 
Scriven, M. [1959]: 'Explanation and Prediction in Evolutionary Theory: Satisfactory Explanation of the Past Is Possible Even When Prediction of the Future Is Impossible', Science, 130, pp. 47782.

Sharp, G. C., Schellhas, L., Richardson, S. S. and Lawlor, D. A. [2019]: 'Time to Cut the Cord: Recognizing and Addressing the Imbalance of DOHaD Research towards the Study of Maternal Pregnancy Exposures', Journal of Developmental Origins of Health and Disease, 10, pp. 50912.

Simpson, S. J. and Raubenheimer, D. [2012]: 'The Nature of Nutrition: A Unifying Framework from Animal Adaptation to Human Obesity', Princeton and Oxford: Princeton University Press.

Solon-Biet, S. M., Walters, K. A., Simanainen, U. K., McMahon, A. C., Ruohonen, K., Ballard, J. W. O., Raubenheimer, D., Handelsman, D. J., Le Couteur, D. G. and Simpson, S. J. [2015]: 'Macronutrient Balance, Reproductive Function, and Lifespan in Aging Mice', Proceedings of the National Academy of Sciences, 112, pp. 3481-6.

Stearns, S. C. [2015]: 'Evolution and Medicine (2015) with Stephen Stearns Lecture 8.1 - Mismatch: Introduction', YouTube, <https://youtu.be/07i6lyNMeKk>.

Stearns, S. C. and Medzhitov, R. [2015]: 'Evolutionary Medicine', 1 edition., Sunderland, Massachussetts: Sinauer Associates is an imprint of Oxford University Press.

Tanaka, M. M., Godfrey-Smith, P. and Kerr, B. [2020]: 'The Dual Landscape Model of Adaptation and Niche Construction', Philosophy of Science, 87, pp. 478-98.

Williams, G. C. and Nesse, R. M. [1991]: 'The Dawn of Darwinian Medicine', The Quarterly Review of Biology, 66, pp. 1-22. 